\title{
A Study on the Automatic Sensing Device for Gas Leakage of Cooling Plate Using the Microprocessor System
}

\author{
Jee-Seok Wang ${ }^{1} \cdot$ Hee-Jong Yoon ${ }^{2} \cdot$ Ki-Seong $\mathrm{Kang}^{2} \cdot$ Jong-Do Kim ${ }^{\dagger}$ \\ (Received April 11, 2011; Revised May 18, 2011; Accepted May 21, 2011)
}

\begin{abstract}
The cooling water circulation plates had been used to drop the temperature of refractory outside shell of common cooling system by using cooling plate or stave type. When they are attacked by surrounding gas, they are corroded and the water flows in the refractory due to leakage of water. So, the life of refractory material is shortened and changed due to the worse conditions of cooling system. The automatic sensing device for water leakage of cooling plate is developed to check the position of trouble by using the microprocessor system when cooling water leak and gas are flowed into the cooling plate through the leakage position. The flowed gas is detected in the micro-process system which delivers the detected position of cooling plate or stave to main control room through the wireless-radio relay station. This system can be possible to detect the position of cooling plate or stave against the water leakage part immediately and then deliver the signal to main control room by using the microprocessor system and wireless-radio relay station. This system will be developed in changing the working condition from manual system to unmanned auto alarm system.
\end{abstract}

Key words : Cooling system, Micro-process, Cooling plate, Stave

\section{Introduction}

New cooling system became more refined and improved. New techniques were introduced and working environment and worker's rights became issue at hand.

In case of blast furnace the iron ore and coke go through a chemical reaction and produce a large quantity of gas. The gas gets dissolved in cooling water and flows out of the system. Then, the gas and the water get separated when the fluid passes through the gas collection device. Gas mixed with cooling water remains in upper part of the fluid and flows through the passage. Using the characteristics of the liquid stopped at places of irregularity, chamber-like gas collection device is installed to collect gas. If the leakage of gas dissolved in the cooling water flowing inside the cooling plate is not pre-detected, it can cause crack or damage to the common cooling system, including the device and inner wall and eventually it can be detrimental to the safety of the working environment. Many researches are made to prevent gas leakage in common cooling system. Solving this potential hazard is viewed as an improvement and invention of new technology to the steel industry. This research aims to improve quality of

\footnotetext{
† Corresponding Author (Division of Marine Engineering, Korea Maritime University, E-mail:jdkim@hhu.ac.kr, Tel: 051-410-4253)

1 Division of Mechanical and Information Engineering, Korea Maritime University

2 Department of Research and Development, Hazon Co., Ltd
} 
steel and work environment and to remove any safety hazard by developing automatic safety device against gas leakage in common cooling system using microprocessor [1]. This system automatically detects any gas leakage and then delivers the signal to main control room via the microprocessor system and wireless-radio relay station, allowing the operator to monitor and detect any leakage.

\section{Experiment and Method}

\subsection{Test Protocols}

The cooling pipe is connected to gas collection device to collect gas dissolved in cooling water. At the upper part of the device, detection device for cooling system, consisting of gas detection device and level detection device is installed. Electronic gas collection group that detects gas occurring from cooling device consists of electric main board device which detects all input signals and provides a junction role. The one side of the device above is connected to the gas collection device and the other side is connected to the water pipe. This device provides the electronic gas collection and detection device that detects gas from cooling

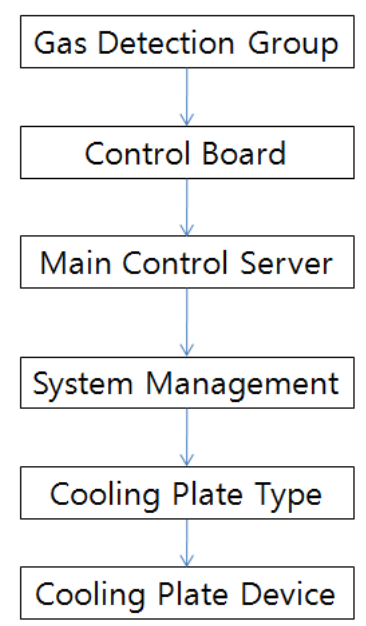

Figure 1: Fabrication process of automatic sensing device for water leakage of cooling pipe at common cooling system facility located at upper part of gas collection device. This gas is dissolved in the cooling water and moves through the system and finally becomes separated when the liquid passes through the gas collection device. Due to the portion of gas and water, the gas stays at upper part of the cooling water. Using the characteristic which the gas becomes dormant when it moves in irregular area, chamber-like gas collection system is installed at water passage, allowing the gas to be collected at the gas collection device.

Figure 2 explains the process of cooling plate-type detection device and stave-type device for automatic detection device of cooling pipe leakage.

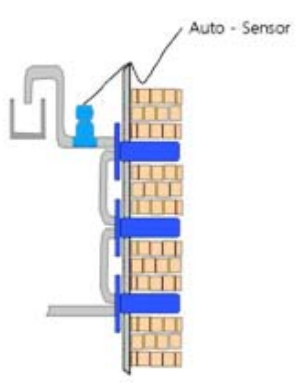

a) Cooling Plate-Type

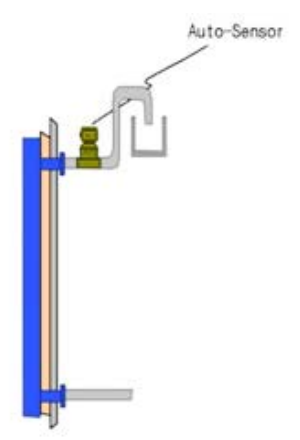

b) Stave-Type
Figure 2: Automatic sensing device types for gas leakage of cooling pipe at cooling system

Gas collection device is designed to be wider than the usual water pipe, allowing the fluid to be slowed and decreased in pressure. This allows the gas to float easier. Vortex flow prevention plate is installed to prevent vortex flow of cooling water [2]. It is porous plate that prevents vortex flow of cooling water, allows the dissolved gas to rise near the upper part of fluid, makes in-and-out passage of liquid efficient, minimizes gas loss, and makes gas collection device more effective. Figure 3 explains the internal structure for unmanned detection device for gas leakage. 


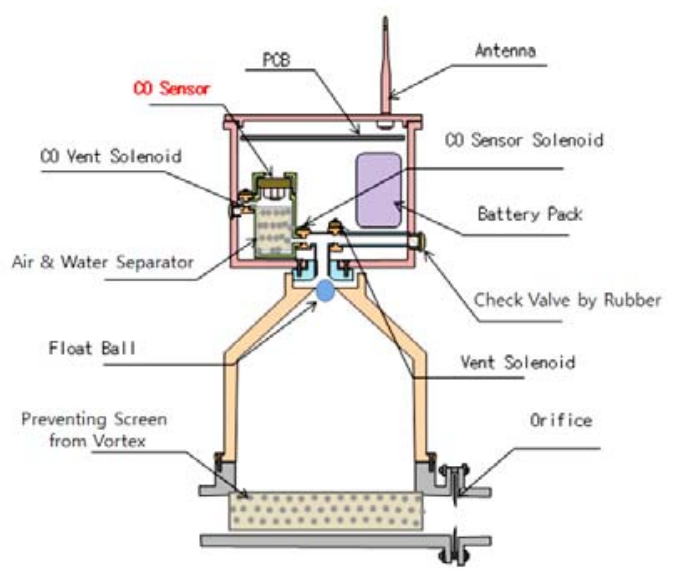

Figure 3: Internal structures of automatic sensing device for gas leakage of cooling pipe at gas cooling system

\subsection{Analysis on electronic detection device for gas leakage}

The gas detection group of unmanned detection device consists of the following: replacement group that exchanges power and sensor for detection device, control port device for electronic solenoid valve that automatically opens when gas is collected, level detection device that sends the signal to the control port device, relay device that sends the detected signal to the main server, main control server and display device that stores the signal, and wireless communication device that sends the main control server stored data to central monitoring facility [3].

And also the gas detection group contains replacement device that replaces power and detection group, electronic solenoid valve control port device that automatically opens the valve when gas is collected, and level detection supplementary device that automatically opens the valve and detects the gas level added.

Figure 4 displays the automatic detection device and Figure $\mathbf{5}$ is an appearance photo of gas leakage device.

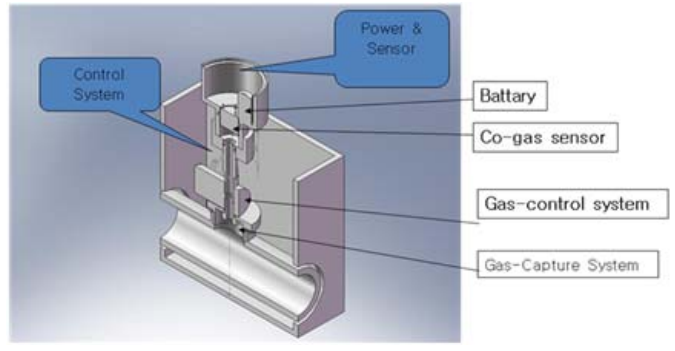

Figure 4: Automatic detection device gas leakage electronics device of cooling pipe at cooling system

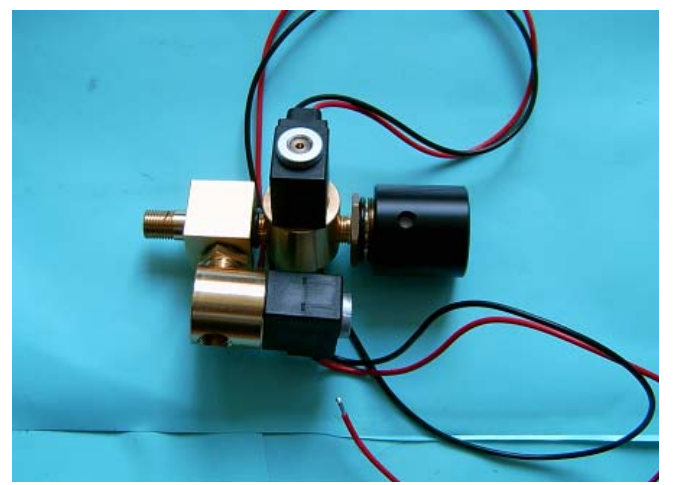

Figure 5: Appearance photo of gas leakage electronics device

\section{Results and Inquiry}

In this research, automatic sensing system for gas leakage collects the gas in cooling system of the gas collection device. The system works when it detects the level of gas decline with the amount of gas present, and the level detector senses the lowered level, and thus activating the electronic sensor [4]. Gas detection sensor detects the gas activates the electronic solenoid valve allowing the gas to flow towards the gas detection sensor and gas collection device. The gas collection device is designed in triangular pyramid shape, making the device wider than the cooling water pipe. This allows the gas to move faster. To make replacement and repair, upper part of detection device is designed to be detachable. if the upper part is turned clockwise or counter-clockwise, upper groove and lower part can be detached. 


\subsection{Main Control Server for Unmanned detection device}

A numerous number of tuyere or cooling plate is installed based on the size of the cooling system. They are placed in the location of high complexity inside the pipes or location of cooling plates. These devices are mainly installed at places of high heat, dust, gas and etc. To allow the device to be unmanned, the system must go through careful comparison with previous system. This comparison is done with device that alerts the location of error occurring from the cooling device in timely manner [5]. Common cooling system should be designed to allow cooling water to circulate and cooling plates to be installed, thus making the facility protected. The previous analogue method should be compared with new digital and wireless method. Figure 6 describes the main control device, using wireless network and ISM wireless communication method.

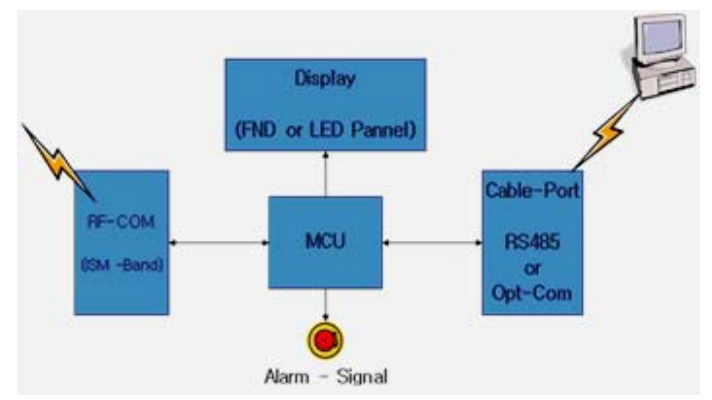

Figure 6: Configuration of main control device

\subsection{Comparing the monitoring system based on signal transfer}

Early detection of the damage at cooling plate provides a safety system by preventing gas leakage of cooling system, and ultimately contributes to the advancement of steel industry and improvement of new technologies. This research aims to improve the quality of steel products and create better and safer working environment by making gas leakage safety device automatic and providing a digital solution distinct from previous manual method.
Previous models of using man power to detect leakage caused lesser accuracy, working hazard and most of all, detrimental effect to workers' health due to gas toxication and exposure to dust.

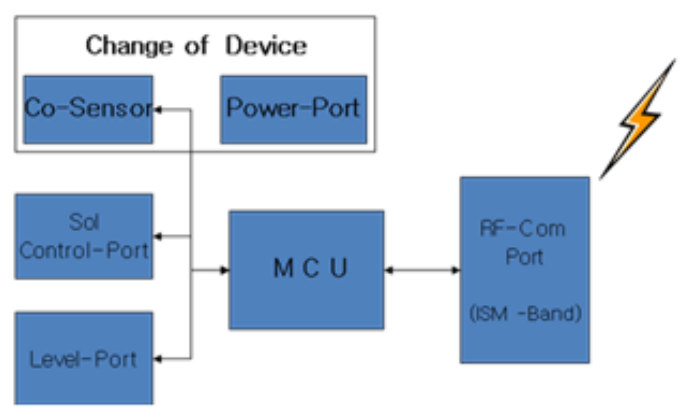

Figure 7: Configuration of main control board

Figure 7 describes a control board device that controls the detected signal automatically. This system allows the sensor to be replaced at the power and digitizes the analogue signal, creating monitor comparison method using wireless communication.

\subsection{Comparing Monitoring system using ZIGBEE - NETWORK}

Automatic gas leakage safety device detects all gas leakage automatically, transfers relevant signal to relay device and then sends the signal to main center, allowing the system to display the occurring event [6].

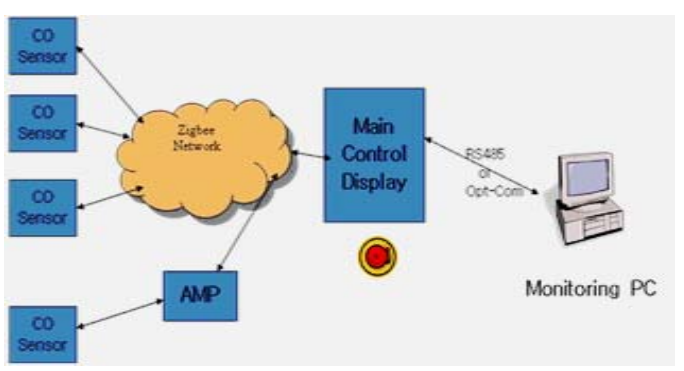

Figure 8: Operating system of main control monitoring device 
Figure 8 describes a signal transferred through ZIGBEE network to the main control server. The signal is displayed and then monitored through RS 485 or optical cable. Depending on the conditions, signals sometimes get transferred via relay device. This digital process is discrete from previous analogue type in its relay method. Figure 9 and Figure 10 shown the comparing monitoring results of automatic sensing device, which show the

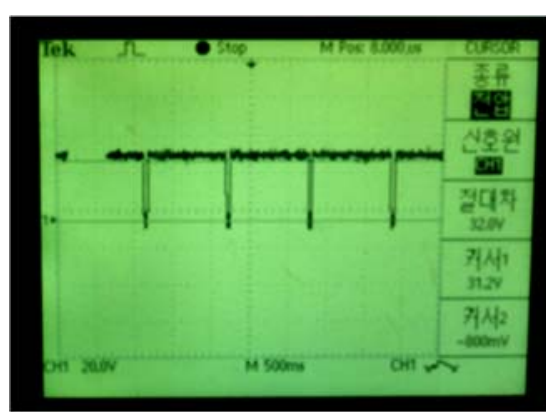

Figure 9: Before gas level detection

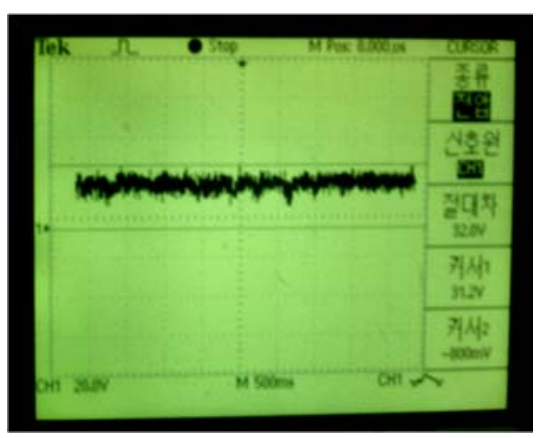

Figure 10: After gas level detection

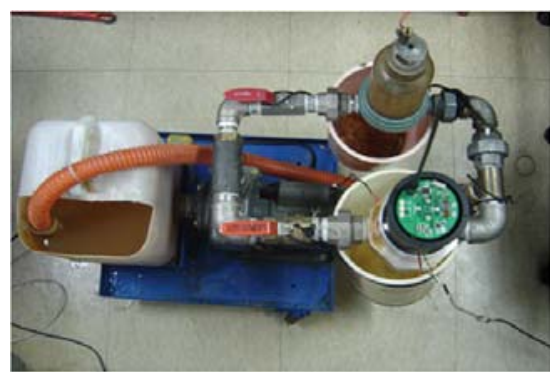

Figure 11: Appearance photo of gas leakage system relation between the before and after level detection Form them, we can show the different peaks of the detected gas level at monitoring system. Figure 11 shows the appearance photo of gas leakage system used as equipment of Measurement system. Figure 12 shows the detected gas level at monitoring system flow chart. Figure $\mathbf{1 3}$ show the detection system flow chart.

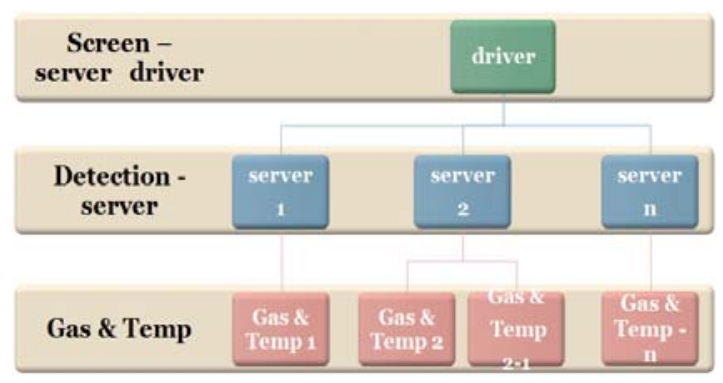

Figure 12: Gas level monitoring system flow chart

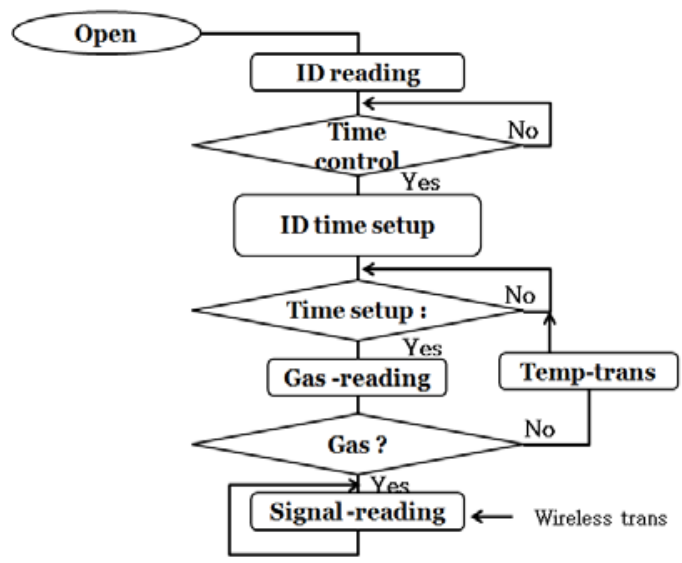

Figure 13: Gas detection server system flow chart

\section{Conclusion}

This research is on the development of unmanned automatic gas detection device at common cooling system.

To provide accurate information on damage to cooling system and tuyere, the device is designed as independent and sealed type, allowing the device unaffected of external factors. Using the automatic electronic detection system, the detection device 
provides accurate information on the occurring events or damage. This device improves the previous inaccurate method of using man power or manual gas collection method and thus saves time and man power. Moreover, the device allows environment-friendly working environment, provides more competitiveness and has other benefits. This research achieved the following economical results.

1) Development of an unmanned automatic gas leakage detection device

- Electronic gas collection and detection technology electronically connecting gas collection device to gas detection device, and level detection device.

2) Development of main control server for detected signal and supplementary display device

- Main control server and display device that stores the detected signal

3) System operation design technology that transfers and monitors the signal.

- Wireless communication device that transfers the stored data from main control server to central monitor facility and system design technology for safely controlling the unmanned detection device for cooling device inside the common cooling system..

\section{References}

[1] E. Chanran, B. Maton and A. Four Ment, "Effect of Postural Muscle Fatigue on the Relation Between Segmental Posture and Movement”, J. Electromyography and Kinesiology , vol. 12, pp. 67-69, 2002.

[2] Vitasalo JHT, et al., "Signal Characteristics of EMG during Fatigue”, Eur J Appl.Physiol, vol. 37, pp. 111-121, 1977.

[3] C.J.De Luca, "The Use of Surface Electromyography in Biomechanics”, J. Appl. Physiol. vol. 13, No. 2. pp. 135-163, 1997.

[4] The Freedonia Group, Inc., Power Supplies for Portable Products to 2008, Pub Date, 2004.
[5] H.L. Garner, "Number systems and arithmetic", Advenced in Computers, vol. 6, pp. 131-194, 1975.

[6] S. Bahng, Y. Park and J. Kim, "QR-LRL signal detection for spatially multiplexed MIMO system,” IEICE TRANS. Commun., vol. E91-B, No.10, pp. 3383-3386, 2008.

\section{Author Profile}

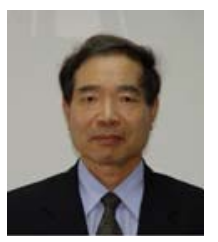

\section{Jee-Seok Wang}

He received his B.S. degree in Marine Engineering from Korea Maritime University in 1971. He then received the M.Eng. and Ph.D degrees in master of Engineering from nantes University in France in 1975 and 1979. Since 1973, he has been a professor of Mechanical Information Engineering Department of Korea Maritime University since 1973.

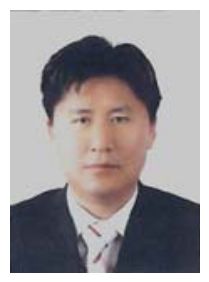

\section{Hee-Jong Yoon}

He received his B.S. degree in Marine Engineering from Korea Maritime University in 1985. He then received the M.Eng.in master of Engineering from Korea Maritime University in 2004 and entered the doctor course of the university Korea Maritime University in 2006, and President of Hazon Co., Ltd. since 2009.

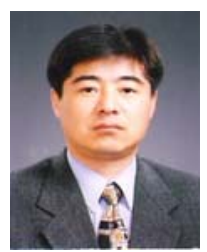

\section{Ki-Seong Kang}

He received his B.S. degree in electronic Engineering from kwon dong University in 1986. He then received the M.Eng. and Ph.D degrees in Electron Engineering from Myung Ji University in 1988 and 1995. From 1989 to 1995, and chief of R\&D Center of Hazon Co.,Ltd since 2009.

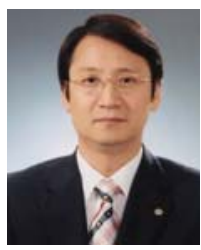

\section{Jong-Do Kim}

He received his B.S. degree in Marine Engineering from Korea Maritime University in 1985. He then received the M.Eng. and Ph.D degrees in Laser Welding Engineering from Osaka University in 1995 and 1997. From 1997 to 1998, he was a Research Fellow in JWRI of Osaka University. Since 1998, he has been a professor of Division of Marine Engineering at Korea Maritime University. His research interests are in Laser Precision Processing and Its Automation. 